\title{
STUDY OF NOVEL POTASSIUM PERMANGANATE STAINING IN COMPARISON WITH CONVENTIONAL ZN STAINING FOR THE DIAGNOSIS OF PULMONARY TUBERCULOSIS
}

\author{
Banu A, Anand T \\ Department of Microbiology, BMC \& RI, Bangalore, India.
}

\begin{abstract}
An estimated 1.8 billion individuals are infected worldwide with the Tuberculosis and there is one death due to it every 15 minutes. It is essential that a fast, effective and inexpensive diagnostic test for TB is developed as early diagnosis stands central to the control of the disease. Traditional diagnosis of tuberculosis is still dependent on sputum microscopy by ZN stain that requires expertise and lacks sensitivity. In this study, we tried to evaluate the use of potassium permanganate staining which is easy, inexpensive and does not require much expertise for screening. Potassium permanganate oxidises Mycolic acid present in cell wall of Mycobacterium sp. which evolves an identifiable oxidation pattern. This study was undertaken to investigate this property of potassium permanganate as a typical laboratory test bridging the gap between sensitivity and time restraint required for an early diagnosis of tuberculosis. The sputum samples (early morning - spot) were collected from patients. From each sample two slides were prepared; one was stained with ZN stain and other with modified potassium permanganate staining. Sputum samples were simultaneously cultured on LJ media after decontamination and concentration. The pattern evolved on staining with potassium permanganate was observed under light microscope and assessed as a diagnostic tool. We found that the pattern formed on oxidation was in association with the AFB status of the sputum $(p=0.0001)$.
\end{abstract}

Keywords : Acid- Fast bacilli; Mycolic acid; Potassium permanganate oxidation.

\section{INTRODUCTION}

Worldwide increase in tuberculosis is indicative of our failure to use modern medical advances to control an ancient enemy. Despite killing 2 million people every year, TB is arguably the most neglected disease. India ranks number 11 and constitute nearly $30 \%$ of the global TB burden. Co-infection of TB with HIV-AIDS and the emergence of multidrug-resistant tuberculosis (MDRTB) have brought tuberculosis back into the priority list of the health care providers globally. It is essential that a fast, effective and inexpensive

\author{
Correspondence to \\ Dr. Asima Banu \\ Associate Professor \\ Department of Microbiology \\ BMC \& RI \\ Bangalore, India \\ E-mail: asima.banu@gmail.com
}

diagnostic test for TB is developed as early diagnosis stands central to the control of the disease.

The diagnosis of TB traditionally depends on demonstration of tubercle bacilli in clinical specimen. Direct smear examination after ZN stain is a simple and rapid test, but it lacks sensitivity (detects $>10^{4}$ bacilli/ml). This continued dependence on more than hundred years old smear test which has a sensitivity of $50 \%$ and is compounded by its inability to diagnose drug resistant bacilli. Conventional culture methods using LJ medium, despite being more sensitive ${ }^{1}$ (detects 10-100 bacilli/ml) is time consuming (3 to 12 weeks), and the modified culture system, BACTEC, takes 7-12 days. Advances in the field of molecular biology have provided rapid diagnostic tools such as PCR based on nucleic acid amplification with reduced turnover time for the unavailability in peripheral centers, cost ineffectiveness and the requirement for specialist personnel has limited its use. 
The unique cell wall structure of Mycobacterium tuberculosis accounts for its unusual low permeability and resistance towards common antibiotics. The main structural element consists of a cross-linked network of peptidoglycan in which some of the muramic acid residues are replaced with arabinogalactan, which in turn is acylated at its distal end with mycolic acids. The entire complex is essential for viability in $M$. tuberculosis and other Mycobacteria. ${ }^{2}$

Potassium permanganate oxidises Mycolic acid present in cell wall of Mycobacterium sp. which evolves an identifiable oxidation pattern. This study was undertaken to investigate this property of potassium permanganate as a typical laboratory test bridging the gap between sensitivity and time restraint required for an early diagnosis of tuberculosis.

\section{METHODOLOGY}

This prospective study was done to evaluate the use of the above stated method as a diagnostic tool for tuberculosis. This short term research project was conducted in Department of Microbiology, Bowring \& Lady Curzon Hospital, Bangalore Medical College and Research Institute, Bangalore over a period of three months (June - August 2009). We included patients attending RNTCP centers in Victoria and Bowring Hospitals, Bangalore with clinical presentation indicative of tuberculosis (like chronic cough $>3$ weeks, haemoptysis, etc) who were not on ATT to take part in the study with an informed consent. The sputum sample was collected from the RNTCP centers, where the treating team routinely uses sputum samples for diagnostic purpose. On the basis of ZN staining results, 50 samples were collected, of which 25 were AFB positive and 25 were AFB negative according to RNTCP criteria. $^{3}$ The ratio of AFB positive to AFB negative samples was deliberately kept $1: 1$ to have an ample opportunity to investigate AFB positive samples.

Processing of the samples: The selected samples were transported to the laboratory and processed on that very day itself. All the samples were handled carefully in a bio safety cabinet (Class II). Three parts were prepared from each specimen, one used for the $\mathrm{ZN}$ technique ${ }^{4}$, one for potassium permanganate stain; and the other for culture inoculation. All the samples were cultured on LJ media using standard precautions and were incubated for 8 weeks and the culture positives were identified using standard techniques. $^{5}$

Potassium permanganate (PP) staining technique:

1) One $\mathrm{ml}$ of sputum sample was centrifuged at $2000 \mathrm{rpm}$ for 5 minutes and the supernatant was discarded.

2) The pellet is washed once with $500 \mu l$ sterile water and again centrifuged at $2000 \mathrm{rpm}$ for 5 minutes.

3) The pellet was suspended in $15 \mu$ l of sterile water. $10 \mu$ of suspension is placed on a microscopic slide and heat fixed.

4) Then $10 \mu$ saturated potassium permanganate solution was added and the slide is incubated at $56^{\circ} \mathrm{C}$ for 5 minutes until dehydrated.

5) The slide is washed by sterile water until excess of color of potassium permanganate is removed.

6) After drying the slide at room temperature, the slide is subjected for microscopic examination.

Statistical analysis: Statistical analysis was performed using the Statistical Package for Social Sciences (version 16.0). The groups were compared using chi-squared test. Calculation of $p$-value was done using Fischer's exact test. Statistical significance was fixed at $p<0.05$.

\section{OBSERVATION AND RESULTS}

The microscopic examination of the smears stained with modified potassium permanganate technique revealed a distinguishable pattern between AFB positive and AFB negative specimen. The positive or negative were strongly supported by results obtained from ZN staining and culture growths. The observations were made under light microscope at a magnification of 10X, 40X and 100X oil immersion objectives.

The salient features of the pattern evolved under observed under oil immersion magnification have been mentioned below:- 
AFB positive smears:

- Deep brown to orange irregular patches seen (of 2-3 mm diameter in size).

- Patches are generally broken into polygons of indefinable shapes and sizes (Honey comb pattern).

- Pattern rests against a translucent background.

- More prominent in the centre of the smears.

$>$ AFB negative smears:

- The above mentioned patches are absent.

- The smear shows a uniformly translucent background with occasional artifacts.

Out of 50 sputum samples, 25 were AFB positive and 25 AFB negative based in the results obtained from ZN staining technique and culture confirmed. The Potassium Permanganate staining successfully picked up all 25 AFB positive. All the other 25 AFB negative samples were also shown negative by this staining technique. The results show a definite association between the pattern formed and the AFB status of sputum sample.

\section{DISCUSSION}

This is a pioneering study demonstrating the recognition of the pattern formed on oxidation of mycolic acid using $\mathrm{KMnO}_{4}$. In the present study, the results of AFB ZN smear and culture on LJ media were analysed in comparison with Potassium Permanganate stain from 50 cases of clinical suspicion of pulmonary tuberculosis. In the study, 25(50\%) cases were culture positive. ZN stain was positive in $24(48 \%)$ cases, whereas Potassium permanganate stain was positive in $25(50 \%)$ culture positive cases. The possible reason of 1 case being negative by ZN stain is that the samples stained with potassium permanganate were centrifuged prior to it. This would increase the bacterial concentration in the sample and possibly increase the sensitivity of the test. Ellena $M$ Peterson et al ${ }^{(6)}$ have reported that concentration smears of AFB have increased the sensitivity to $91 \%$ as compared to $81 \%$ of direct smears.

The comparison of the results obtained on PP staining with the AFB status of sputum samples reveals a definite association between the two where potassium permanganate staining was positive in all the $\mathrm{ZN}$ positive smears. Since no data was available about a similar study, no comparisons can be made.

The major difficulty in staining tubercle bacilli arises because their surface is coated with an unsaponifiable waxy substance. For such bacilli, the success of conventional staining techniques depend on the ability of the dye to uniformly penetrate the cell wall through this waxy barrier, while leaving intact the acid fast character of the bacilli (in ZN staining technique, heating with conc. carbol fuschin). In this novel potassium permanganate staining technique, the whole cell wall of bacilli is oxidised using a saturated solution of potassium permanganate. In turn, mycolic acid, present in the cell wall, is oxidised to evolve 'honey comb pattern'.

This test avoids the complexity involved in other methods and presents an easier method. The pattern evolved in AFB positive samples is distinguishable and could be successfully employed for diagnosis of tuberculosis. It is easier for the eye to detect brown honeycomb pattern rather than a red fuschin stained bacillus in of surrounding. The time required by PP staining before declaring smear as negative was 2 minutes compared to 8 minutes with ZN staining ${ }^{(7)}$. It's a fast test and is highly cost effective at the same time as the only reagent required is potassium permanganate. Moreover the expertise required in handling reagents is less and reading results is easier.

The possible application of this test lies in its potential to be used as a mass diagnostic modality as it is easier, cost effective, and takes the minimal time. This makes it aptly suited for the primary health centers in a developing country as ours. It could also be used to funnel down the patients being investigated for tuberculosis.

This study has many strength. The AFB positive samples suggested by ZN staining technique were confirmed by culturing them on LJ media. ZN staining lacks sensitivity, the sputum samples with a bacterial load of less than $10^{4}$ bacilli/ml are not detectable ${ }^{(1)}$. Culturing the samples picks up even those which have a bacterial load of 10-100 bacilli/ml, much more sensitive than $\mathrm{ZN}$ staining alone. A sincere attempt 
was made to standardise the method to reduce the possible error that may have crept in while the experiments.

Certain limitations and caveats should be kept in mind while interpreting the results of this study. Firstly, sensitivity and specificity of the method has not been evaluated. The sample size is too small as still it is an ongoing study.

\section{CONCLUSION}

The microscopic examination of the smears stained with modified potassium permanganate technique revealed a distinguishable pattern between AFB positive and AFB negative specimen. This implies a possible application of potassium permanganate staining as a method in diagnosis of tuberculosis.

\section{FUTURE PROSPECTIVES OF THE STUDY}

- The sensitivity and specificity of the method has to be estimated, and compared with the standard ZN staining technique being used by including more subjects.

- The mechanism of oxidation and the products hence formed needs to be looked into. Colour tests based on the detection of product formed could be the shape of the things to come in future.

\section{REFERENCES}

1. Mridula Bose, Editorial. The Archetype of Laboratory Diagnosis in Tuberculosis - The shape of things to come. Indian J Chest Dis Allied Sci 2006; 48: 167169.
2. Barry et al. (1998). "Mycolic acids: structure, biosynthesis, and physiological functions." Prog. Lipid Res. 37(3): 143-79.

3. Central TB Division, Directorate General of Health services. TB India 2006; RNTCP Status Report. New Delhi: Nirman Bhavan.

4. Mandell GL, Douglas RG Jr, Bennett JE. Princioples and practice of Infectious Diseases. Volume $5^{\text {th }}$ ed. Churchill Livingstone, New York; 2000.

5. Collee JG, Marmion BP, Fraser AG. Tests for the identification of bacteria. Mackie and Mc Cartney Practical Medical Microbiology. 14 $4^{\text {th }}$ ed. Churchill Livingstone; 1996. p 131-50.

6. Soumya Swaminathan, Sangeeta M Arun Kumar N, Menon PA, Bena Thomas, Shibi K, et al. Pulmonary tuberculosis in HIV positive individuals. Preliminary report on clinical features and response to treatment. Ind J. Tub 2002, 49:189.

7. BaF Reider HL. A comparison of Fluorescence microscopy with $\mathrm{ZN}$ technique in examination of sputum for AFB. Int J Tuberc Lung Dis. 1999; 3(12); 1101-5.

8. Sayed Mohsen Khatami. The Oxidative Reaction of Potassium Permanganate with Mycolic Acids Leads to a Unique Diagnostic Pattern for Mycobacterium Tuberculosis. Nature Precedings : hdl:10101/ npre.2008.2365.1 : Posted 3 Oct 2008.

9. Minnikin, D. E. The Biology of the Mycobacteria. Vol, eds C. Ratledge and J. Standford. Academic Press, London, 1982, pp. 95-184.

10. Takayama, Wang, Besra. Pathway to synthesis and processing of mycolic acids in Mycobacterium tuberculosis. Clin. Microbiol. Rev. 2005; 18(1): 81101. 